\title{
Measurement Brownian Dimension of Von Koch Curve
}

\author{
Mahasin Thabit Younis \\ mahasinthabit@uomosul.edu.iq \\ College of Computer Sciences and Mathematics \\ University of Mosul, Mosul, Iraq
}

Received on: 26/02/2018

Accepted on: 20/05/2018

\begin{abstract}
The aim of this paper, it's calculate Brownian dimension of fractal pattern has self similarity (Von Koch Curve). This method is Random Middle Third Displacement in $[0,1]$ has Gaussian distribution. Random processes are the main focus of research by analyzing dynamical systems to determine these 'chaotic' systems function. One such dynamical system is Brownian Motion. Basis of current definitions of physical phenomena.
\end{abstract}

Keywords: Brownian dimension, Brownian Motion, Fractal Sets.

$$
\begin{aligned}
& \text { قياس البعد البراوني لمنحني فون كوخ } \\
& \text { محاسن ثابت يونس } \\
& \text { كلية علوم الحاسوب واليراضيات } \\
& \text { جامعة الموصل، الموصل، العرق علوات ولريلات }
\end{aligned}
$$

تاريخ قبول البحث: 2018105120

تاريخ استلام البحث: 2018102126

\section{الملخص}

إن الهدف من هذا البحث هو حساب البعد البراوني لشكل كسوري يمتلك خاصية التثابه الذاتي وهو منحني فون كوخ. وهي طريقة إزاحة الثلث الأوسط في الفترة [0,1] التي تتبع توزيع كاوس بالاعتماد على العمليات

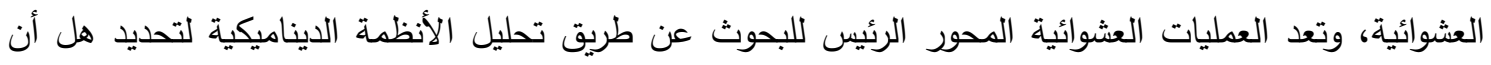

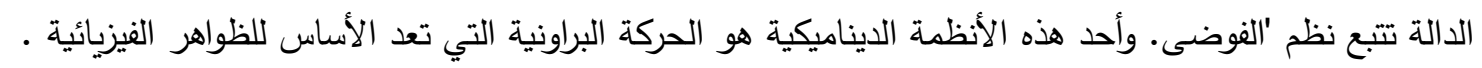
الكلمات المفتاحية: البعد البراوني، الحركة البراونية، المجموعات الكسورية. 1

فأصبحت "الكسوريات"، تلعب دوراً رئيسياً في العديد من التطبيقات المهمة للحركة البراونية. يعد العالم Benoit B. Mandelbrot أول من قدم مصطلح "Fractal" ، ويمكن تعريف الكسوريات بصورة مبسطة بأنها عبارة عن نمط هندسي متكرر على مختلف المقاييس، وهناك العديد من صور الكسوريات في العالم الحقيقي التي

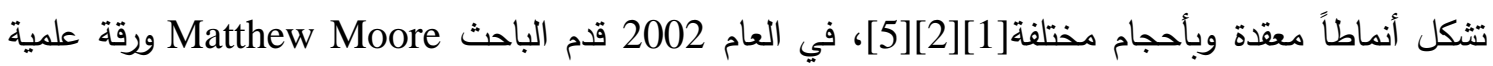
لحساب البعد البراوني لفترة [0,1] باستخدام طريقة المنتصف Middle Point وحساب البعد البراوني للفترة

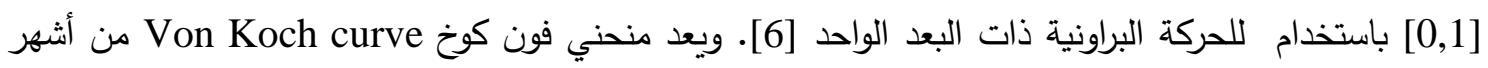
الكسوريات التي تتولد بعمليات تكرارية بسيطة الذي سيكون محور بحثنا هذا والمبين في الثكل(1). 

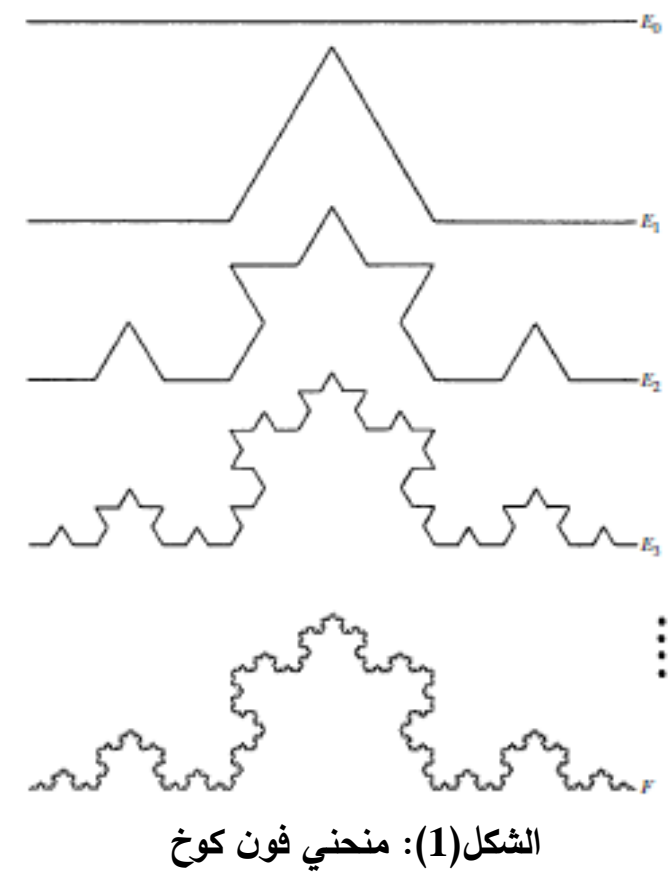

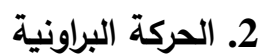

في عام 1827 قدم عالم النبات روبرت براون الحركة البراونية، وهي عبارة عن حركة عشوائية للجزيئات الموجودة في التجاويف داخل حبوب اللقاح في الماء التي يمكن ملاحظتها بالمجهر • وفي عام 1905 نشر العالم ألبرت آينشتاين ورقة علمية فسرت بدقة كيفية حدوث هذه الحركة، وأكدت على وجود الذرات والجزيئات] [7]. والثكل

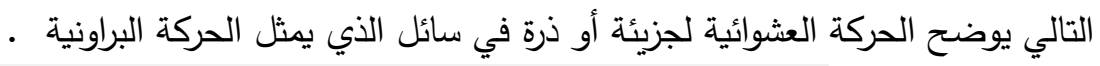
Three dimensional Brownian Motion

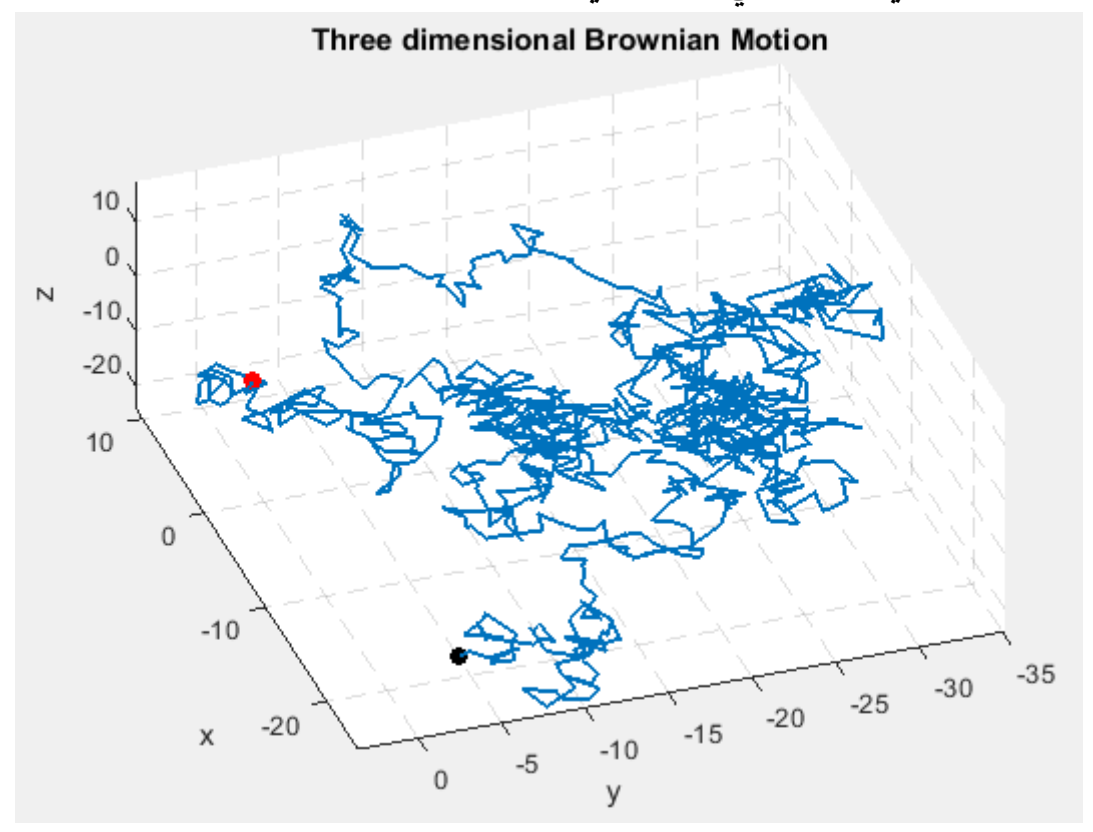

الثكل(2): الحركة البراونية

وتعد الحركة البراونية مغلقة تحت تأثير توزيع كاوس، إذا كان X متغير عشوائي يتبع التوزيع الطبيعي بمعدل وتباين

$$
P\{X>x\}=\frac{1}{\sqrt{2 \pi \sigma^{2}}} \int_{x}^{\infty} e^{\frac{-(u-\mu)^{2}}{2 \sigma^{2}}} d u, \quad \forall x \in R
$$


تسمى العملية الإحصائية لقيمة حقيقية الآتية[9]: $. \mathrm{B}(0)=\mathrm{x}-1$ 2- مقدار الزيادة تكون مستقلة لتغير الزمن هي متغيرات عشوائية مستقلة. $B\left(t_{n-1}\right), B\left(t_{n-1}\right)-B\left(t_{n-2}\right), \ldots, B\left(t_{2}\right)-B\left(t_{1}\right)$ 3- 0 وتباين h.h.

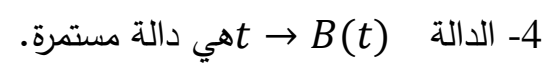

عندئذ يمكننا القول

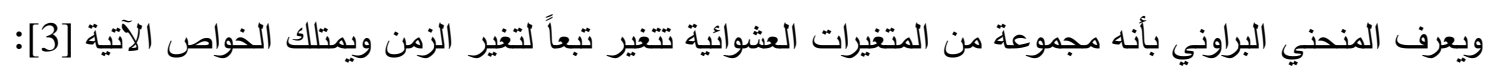

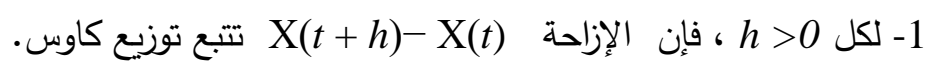

2- الإزاحة الحالية , X(t) Xحيث ان X

$$
\text { السابقة الإها }
$$

3- معدل الإزاحة يساوي صفراً .

3. الحركة البراونية ذات البعد الواحد

يعد أنموذج الحركة البراونية أحد أفضل النماذج المهمة في الميكانيكا الإحصائية، والحركة البراونية هي

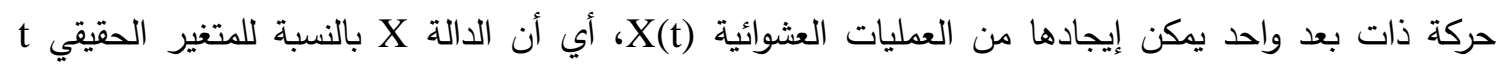
X(t

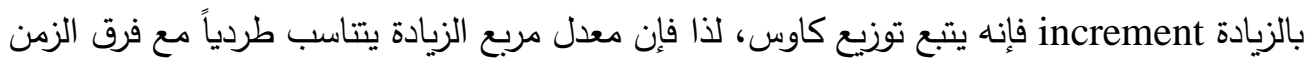
$E\left[\left|X\left(t_{2}\right)-X\left(t_{1}\right)\right|^{2}\right] \propto\left|t_{2}-t_{1}\right|$

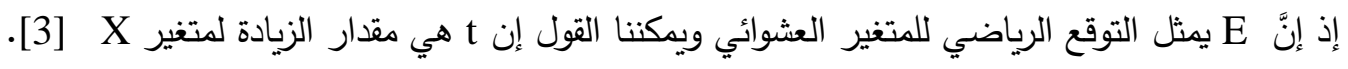

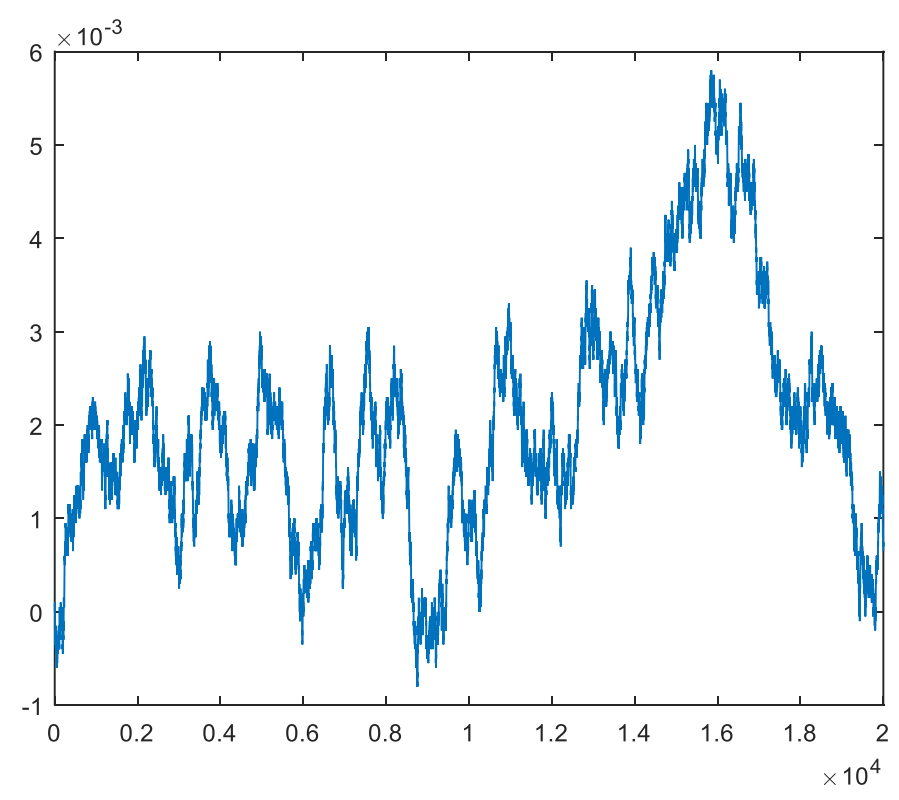

الثكل (3): الحركة البراونية 
Fractional Brownian Motion الحركة البراونية الكسرية

في عام 1940، قدم العالم Kolmogorov الحركة البراونية الكسرية باسم "Wiener spiral" ، وعمل

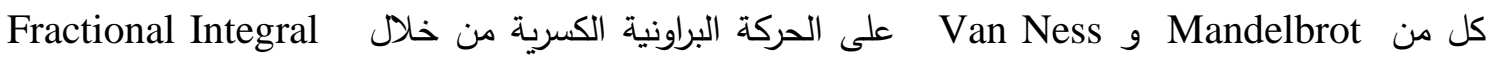
[11]. يستخدم مفهوم الحركة البراونية الكسرية أيضاً في المجال الطبي وخاصة في مجال التصوير بالموجات فوق من فئه الصوتية للكثف عن تشوهات في حجم أو شكل أي من الأجهزة الحيوية. مما يؤدي إلى الكثف عن الأورام

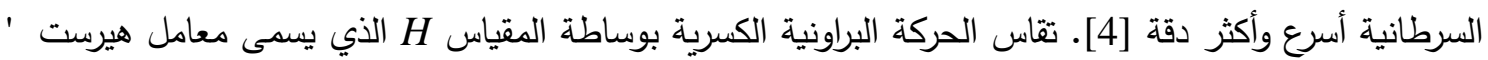

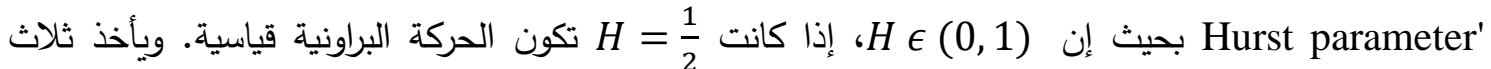
حالات وتبعاً لقيمة H يتغير سلوك الحركة البراونية الكسرية ويمكن قياس البعد البراوني للسطح الذي يكون بعده

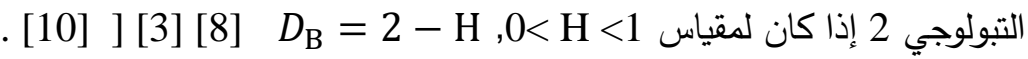
1- إذا كانت $1=0$ ت 2- إذا كانت 3- إذا كانت 24- إذا كانت

Von Koch Curve منحني فون كوخ

يعد منحني فون كوخ أهم هيكل يمكن بناءه في علم الكسوريات بوساطة عمليات تكرارية هندسية بسيطة على الفترة[0, 1]، إذ تقسم الفترة إلى ثلاثة أقسام متساوية ويستبدل الجزء الثاني من الفترة بمثلث متساوي الساقين

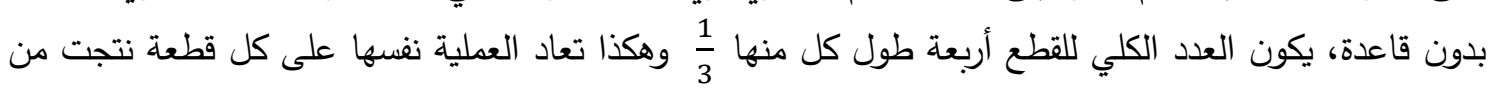
التكرار السابق وكما موضح في الثكل (1). 6. طريقة قياس البعد البراوني لمنحني فون كوخ

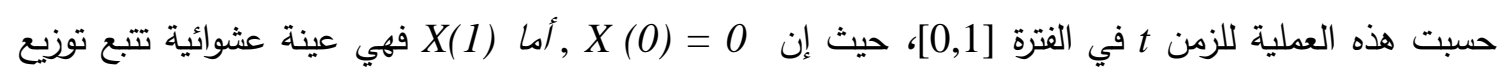
كاوس بمعدل يساوي صفر وتباين $\operatorname{var}(X(1)-X(0))=\sigma^{2}$

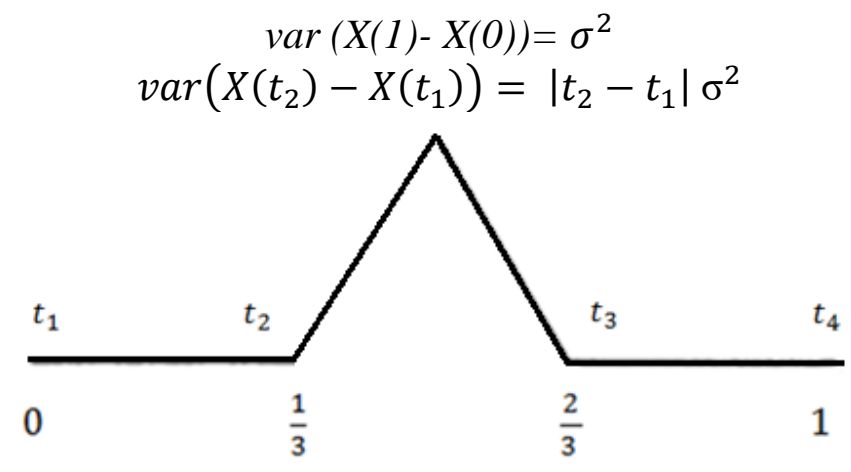

$$
\text { الشكل (4): التكرار الأول لمنحني فون كوخ }
$$

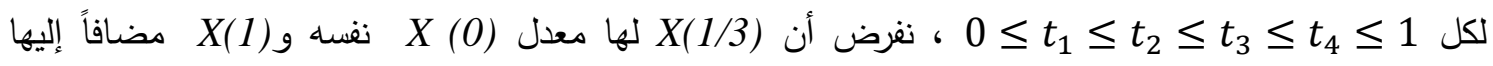

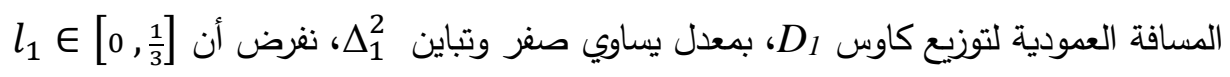
$X\left(\frac{1}{3}\right)-X(0)=\frac{1}{3}(X(1)-X(0))+\mathrm{D}_{1}$ $\operatorname{var}\left(X\left(\frac{1}{3}\right)-X(0)\right)=\frac{1}{9} \operatorname{var}(X(1)-X(0))+\Delta_{1}^{2}$ 


$$
\begin{gathered}
\left|\mathrm{t}_{2}-\mathrm{t}_{1}\right| \sigma^{2}+\Delta_{1}^{2}=\operatorname{var}\left(X\left(t_{2}\right)-X\left(t_{1}\right)\right) \\
\frac{1}{9}|1-0| \sigma^{2}+\Delta_{1}^{2}=\frac{1}{3} \operatorname{var}(X(1)-X(0)) \\
\frac{1}{9} \sigma^{2}+\Delta_{1}^{2}=\frac{1}{3} \sigma^{2}
\end{gathered}
$$

..(1) $\Delta_{1}^{2}=\frac{2}{3^{2}} \sigma^{2}$

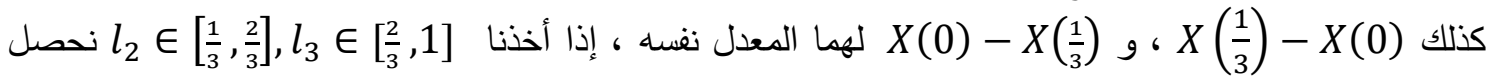

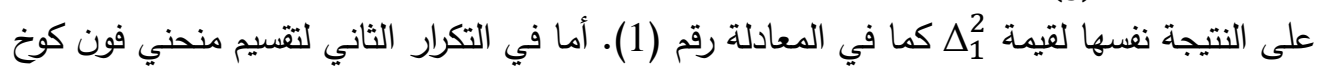

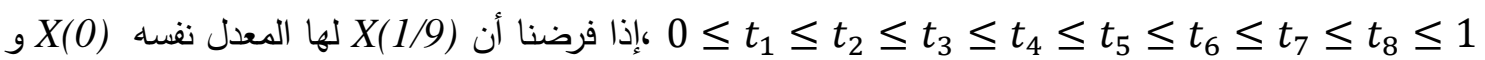

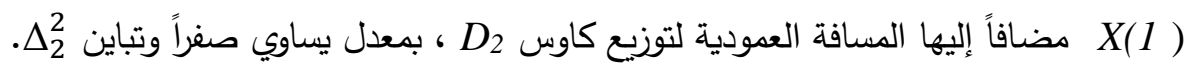

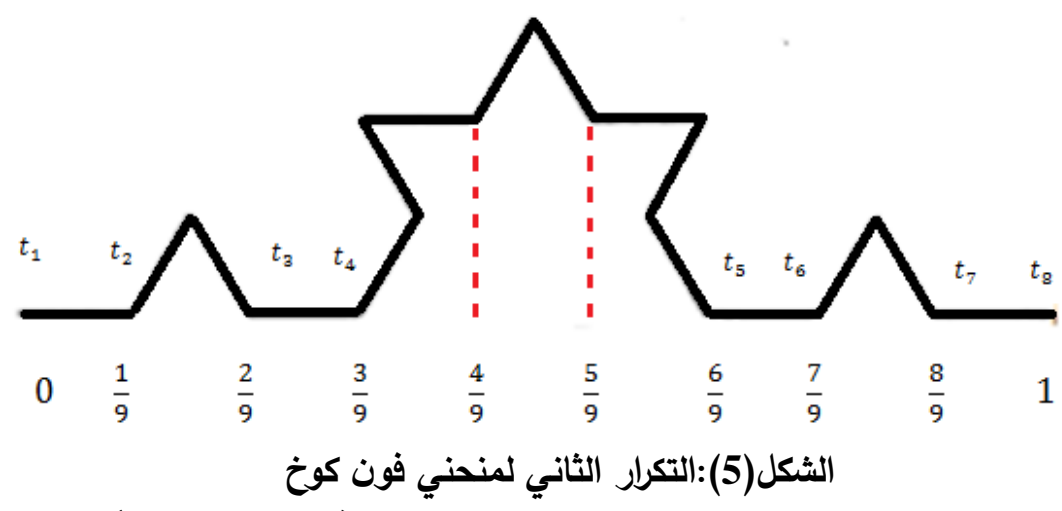

$\mathrm{X}\left(\frac{1}{9}\right)-\mathrm{X}(0)=\frac{1}{3}\left(\mathrm{X}\left(\frac{1}{3}\right)-\mathrm{X}(0)\right)+D_{2}$

$\operatorname{var}\left(X\left(\frac{1}{9}\right)-X(0)\right)=\frac{1}{9} \operatorname{var}\left(X\left(\frac{1}{3}\right)-X(0)\right)+\Delta_{2}^{2}$

$\left|\mathrm{t}_{2}-\mathrm{t}_{1}\right| \sigma^{2}+\Delta_{2}^{2}=\operatorname{var}\left(X\left(t_{2}\right)-X\left(t_{1}\right)\right)$

$\frac{1}{9}\left|\frac{1}{3}-0\right| \sigma^{2}+\Delta_{2}^{2}=\operatorname{var}\left(X\left(\frac{1}{3}\right)-X(0)\right)$

$\frac{1}{27} \sigma^{2}+\Delta_{2}^{2}=\frac{1}{9} \sigma^{2}$

$\Delta_{2}^{2}=\frac{2}{27} \sigma^{2}=\frac{2}{3^{3}} \sigma^{2}$

وبتطبيق الفكرة نفسها على X(2) إلى أن ننتهي من جميع القطع المتكونة من التكرار الثاني فنحصل على قيمة 2ه نفسها وبحساب قيمة التغير في التباين للنحني فون كوخ للتقسيم الثالث والرابع والخامس يمكننا استتتاج $\Delta_{\mathrm{n}}^{2}=\frac{2}{3^{n+1}} \sigma^{2}$ الصيغة العامة لمقدار التغير في التباين فتكون قيمته بالثكل الآتي:

لحساب قيمة H لمنحني فون كوخ لإيجاد سلوك المنحني باستخدام الحركة البراونية الكسرية $\operatorname{var}\left(X\left(t_{2}\right)-X\left(t_{1}\right)\right)=\sigma^{2}\left|t_{2}-t_{1}\right|^{2 \mathrm{H}}$

$\operatorname{var}\left(X\left(\frac{1}{3}\right)-X(0)\right)=\frac{1}{3^{2 \mathrm{H}}} \operatorname{var}(X(1)-X(0))+\Delta_{1}^{2}$

$\left|\mathrm{t}_{2}-\mathrm{t}_{1}\right|^{2 \mathrm{H}} \sigma^{2}+\Delta_{1}^{2}=\operatorname{var}\left(X\left(t_{2}\right)-X\left(t_{1}\right)\right)$

$\frac{1}{9}\left|\frac{1}{3}-0\right|^{2 \mathrm{H}} \sigma^{2}+\Delta_{1}^{2}=\frac{1}{3^{2 \mathrm{H}}} \operatorname{var}(X(1)-X(0))$

$\Delta_{1}^{2}=\frac{\sigma^{2}}{3^{2 \mathrm{H}}}\left(1-3^{-2}\right)$

وتبعاً لتغير الزمن (2.3-n $\left(2.3^{-n}\right.$ وهو قيمة ثابتة بالنسبة لمنحني فون كوخ حسب المعادلات السابقة وبإضافة العناصر العشوائية للتباين التي تكون قيمتها ثابتة أيضاً (2.3 (2.3 نعوض قيمة $\Delta_{1}^{2}$ الناتجة من المعادلة (1) في المعادلة رقم (2) فينتج: 
$\frac{2}{9} \sigma^{2}=\frac{\sigma^{2}}{3^{2 \mathrm{H}}}\left(1-3^{-2}\right)$

$\frac{2}{9} \sigma^{2}=\frac{(8) \sigma^{2}}{(9) 3^{2 \mathrm{H}}} \rightarrow 3^{2 \mathrm{H}}=4$

$2 H=\frac{\ln 4}{\ln 3} \rightarrow 2 H=1.26$ (البعد الكسوري لمنحني فون كوخ)

$H=0.63>\frac{1}{2}$

وتبعاً لقيمة H للمنحني نستتتج أن الإزاحة تكون متزايدة .لذلك فإن البعد البراوني لمنحني فون كوخ

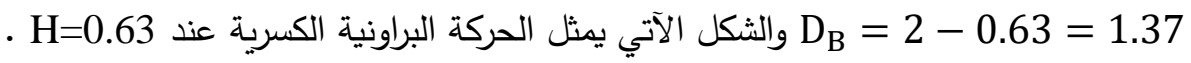

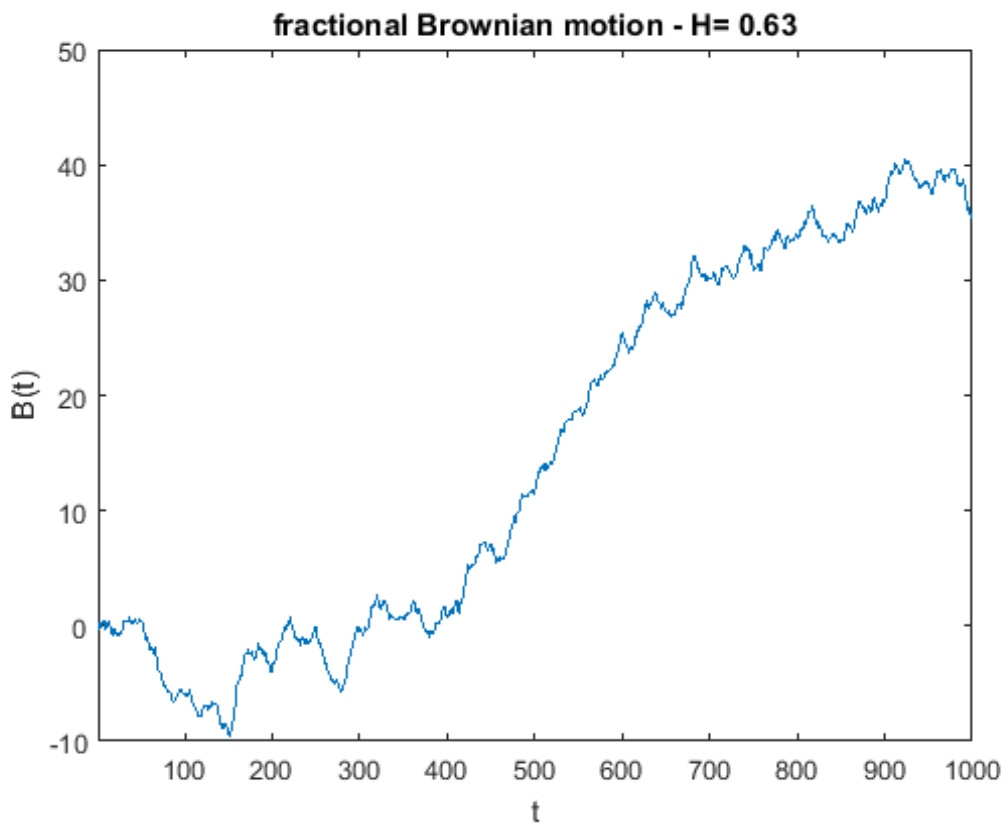

الثكل(6): الحركة البراونية الكسرية

7. الاستنتاجات

ركزنا في هذا البحث على نظام الديناميكية المنفصلة لسلوك منحني فون كوخ باستخدام طريقة جديدة وهي

طريقة إزاحة الثلث الأوسط من الفترة [0,1] التي تتبع توزيع كاوس و إيجاد البعد البراوني له باستخدام عمليات تكرارية على كل قطعة ناتجة من تقسيم المنحني في المستوي الواحد من مستويات التقسيم وإيجاد معادلة عامة

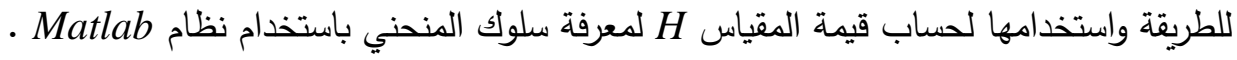




\section{المصادر}

[1] Chinnasamy G. and Vanitha S., 2015," Fractional Brownian Motion and Fractal Analysis of Brain Mri Images ", International Journal of Applied Research Vol. 1, No.3, pp. 21-24.

[2] DeLorto R., 2013,"Fractal dimension and Julia sets", unpublished M. Sc, Eastern Washington University.

[3] Dietmar S., 1988, "algorithm for random fractals', chapter 2"of 'The Science of Fractal Images, Now York.

[4] Jonathan Turner,2012,"Brownian Motion Applied to Human Intersections ", M. Sc Texas State University-San Marcos. Clemson University, Clemson, South Carolina.

[5] Molz F. J. and Liu H. 1997 ,"Fractional Brownian motion and fractional Gaussian noise", water resources research, Vol. 33, No. 10, pp. 2273-2286.

[6] Moore M., 2002,"One Dimensional Brownian Motion" .

[7] Mörters P. and Peres Y., 2003, "Brownian Motion".

[8] Paul Bourgine, 2013,"Hurst Parameter Estimation on Fractional Brownian Motion and Its Application to The Development of The Zebrafish.

[9] Potgieter P., 2007, "The Fractal Geometry of Brownian Motion" UNISA .

[10] Wang R., 2014, " Self-Similar Based Time Series Analysis and Prediction ", unpublished M. Sc, University of Toronto.

[11] Wijeratne C. and Bessaih H. 2015 "Fractional Brownian Motion and an Application to Fluids", Springer International Publishing Switzerland, pp.37-52. 